\title{
EDUCATION
}

\section{Simulated STAT Laboratory in a University Based Medical Laboratory Science Program}

\author{
JANET OJA, JANICE THOMAS, MATTHEW NICHOLAOU
}

\section{ABSTRACT}

A crucial component of Medical Laboratory Science (MLS) student training is clinical experience. This can be delivered through clinical rotations or by clinical laboratory simulation. Weber State University (WSU) in Ogden, Utah utilizes a combination of both to provide clinical experience to MLS students. In response to feedback from bi-yearly meetings with an advisory board, a campus simulated laboratory course was re-designed to enhance student customer service, communication skills, workflow and time management, instrument maintenance and troubleshooting, data entry, and result reporting. The course was modeled after a hospital STAT, or small core, laboratory consisting of blood bank, coagulation studies, chemistry, hematology, urinalysis, microbiology, and specimen processing. The course was taught over two semesters and evaluated by a pre/post survey related to the main educational outcomes, a mock certification exam, and a comprehensive practical exam. The survey results showed that the students' perceived competence increased in all the areas of training, with the greatest increases seen in specimen processing and management and communication. The lowest average score was demonstrated in perceived competence with instrument maintenance. All students achieved a department-required minimum 80 percent competence on the comprehensive practical exam and there was a significant increase $(\mathrm{p}<0.001)$ in mock certification exam scores.

ABBREVIATIONS: MLS - Medical Laboratory Science, WSU - Weber State University, SOPStandard Operating Procedure, LIS - Laboratory Information System, CAP- College of American Pathology, BS- Bachelor of Science, SBAR - Situation, Background, Assessment, Recommend-ation, BOC Board of Certification, LIS - Laboratory Information System, LMS - Learning Management System
INDEX TERMS: Clinical Education, Clinical Simulation, Simulated Laboratory

Clin Lab Sci 2016;29(2):59-65

Janet Oja, MHA, MLS(ASCP) ${ }^{C M}$, Weber State University, Dumke College of Health Professions, Department of Medical Laboratory Science, Ogden, UT

Janice Thomas, MEd, MT(ASCP), Weber State University, Dumke College of Health Professions, Department of Medical Laboratory Science, Ogden, UT

Matthew Nicholaou, DrPH, MT(ASCP), Weber State University, Dumke College of Health Professions, Department of Medical Laboratory Science, Ogden, UT

Address for Correspondence: Janet Oja, MHA, MLS(ASCP) ${ }^{C M}$, Program Director \& Assistant Professor, Weber State University, Dumke College of Health Professions, Department of Medical Laboratory Science, 3875 Stadium Way, Dept. 3905, Ogden, UT 844083905,801-626-7284, janetoja@weber.edu

\section{INTRODUCTION}

Clinical training is a fundamental component of the Medical Laboratory Science (MLS) student's educational preparation. It offers the student an opportunity to observe, practice, and develop hands-on technical, communication, problem-solving, and interpersonal skills in a real workplace setting. ${ }^{1}$ The procurement of sufficient student clinical rotation positions can be challenging for non-hospital based MLS programs. The availability of these spots can also be a limiting factor to student acceptance and graduation rates. ${ }^{2}$

Replicating hands-on experiences in a simulated setting can be a complex undertaking. Weber State University's (WSU) MLS program utilizes a simulated model, along with an abbreviated clinical rotation, to teach and assess clinical skills. The program is assessed using graduate and 


\section{EDUCATION}

employer surveys, certification exam scores, student course evaluations, graduate employment rates, American Society for Clinical Pathology (ASCP) pass rates, and feedback from an advisory board committee.

After meeting with the advisory board some key areas were identified that were lacking in the curriculum. The WSU faculty determined that these areas could be added to an existing simulated practices course (Simulated Laboratory), with the first semester focused in the simulated work environment and the second semester focused on other managerial aspects of medical laboratory sciences such as mock College of American Pathologists (CAP) inspections, Standard Operating Procedures (SOP) writing, job instrument purchasing analysis, instrument validation, and calibration.

\section{MATERIALS AND METHODS}

\section{Course / Laboratory Design}

The initial implementation of the laboratory portion of Simulated Laboratory was used to identify testing, create measurable objectives, make instrument purchasing decisions, identify workflow obstacles, create documents, and design and implement a course survey. The 20 students enrolled were used as a pilot population to help develop the course and were not included in the analysis to follow. They were given the opportunity to be cocollaborators in this process during the weekly scheduled classroom meeting, where student feedback was encouraged.

After reviewing the inaugural Simulated Laboratory course evaluations and workflow concerns, the faculty developed teaching modules for use in the simulated laboratory environment. These modules included customer service, personnel management and effective communication skills, workflow, instrument maintenance and troubleshooting, specimen processing, and data entry and result reporting. Objectives were created for each module, which were then adapted to the simulated laboratory experience. Each week a specific topic or skill was emphasized and objectives were available online via the university's Learning Management System (LMS).

Once per week the students met in a classroom to discuss the previous week's laboratory activities and to work through problems and issues that came up during the laboratory portion. If students missed important information on laboratory requisitions, samples tubes, or any other clerical errors, these were discussed and improvements were made the following week.

The four hundred and eighty five square foot area was divided into chemistry, coagulation studies, hematology, blood bank, microbiology, and urinalysis. A level 2biosafety hood, 55-inch flat screen monitor and a new centrifuge were also added to the new STAT laboratory. The review of the initial semester course also identified the need for a Laboratory Information System (LIS). This concern was addressed by the purchase and implementation of a LIS system through university grant monies.

\section{Study Population}

The 18 students enrolled in the first semester of Simulated Laboratory in the fall of 2013 met once a week to discuss the objectives for the upcoming laboratory session and to discuss the outcomes of the previous laboratory session. These students were in their third or fourth year of the MLS program. Two laboratory sections capped at nine students per section were offered.

\section{Course Evaluation Tools}

The students were asked to complete a twelve question pre-course assessment evaluating their perceived competence regarding specific laboratory related activities such as use of a LIS, performing QC, instrument maintenance, and communication skills (Table 1). Upon conclusion of the first semester, the students then completed the same survey as a postassessment survey.

The students also completed a 100 question mock MLS certification exam. The exam was comprised of questions from several current certification exam review study guides. $^{3,4,5}$ and included questions on chemistry, hematology, microbiology, blood bank, immunology, and body fluids in approximately equal percentages. The same 100-question mock certification exam was repeated upon completion of both semesters of Simulated Laboratory.

The final capstone assessment exercise consisted of students working individually to complete five patient samples involving all of the major disciplines, including QC, specimen processing, and any instrument maintenance necessary within a pre-determined time 
frame of 90 minutes.

\begin{tabular}{|c|c|}
\hline Question (\#, text) & Objective \\
\hline $\begin{array}{l}\text { 1. I feel competent demonstrating } \\
\text { commitment to the values involved in } \\
\text { delivery of quality medical care. }\end{array}$ & Customer Service \\
\hline $\begin{array}{l}\text { 2. I fell competent performing clinical } \\
\text { laboratory tests and procedures in } \\
\text { order to provide accurate diagnostic } \\
\text { data. }\end{array}$ & Customer Service \\
\hline $\begin{array}{l}\text { 3. I feel competent performing quality } \\
\text { control procedures to ensure accuracy } \\
\text { of laboratory data. }\end{array}$ & $\begin{array}{l}\text { Instrument } \\
\text { Maintenance }\end{array}$ \\
\hline $\begin{array}{l}\text { 4. I feel competent performing } \\
\text { preventative and corrective } \\
\text { maintenance on equipment to ensure } \\
\text { quality of work. }\end{array}$ & $\begin{array}{l}\text { Instrument } \\
\text { Maintenance }\end{array}$ \\
\hline $\begin{array}{l}\text { 5. I feel competent accurately } \\
\text { transcribing and recording } \\
\text { information in laboratory documents. }\end{array}$ & Lab Data Entry \\
\hline $\begin{array}{l}\text { 6. I feel competent communicating } \\
\text { results verbally by telephone, by } \\
\text { written report, and by computer } \\
\text { information system. }\end{array}$ & $\begin{array}{l}\text { Management and } \\
\text { Communication }\end{array}$ \\
\hline $\begin{array}{l}\text { 7. I feel competent providing teaching } \\
\text { and education to laboratory students } \\
\text { and other personnel. }\end{array}$ & $\begin{array}{l}\text { Management and } \\
\text { Communication }\end{array}$ \\
\hline $\begin{array}{l}\text { 8. I feel competent interpreting and } \\
\text { acting upon written and verbal } \\
\text { instructions for obtaining specimens. }\end{array}$ & Specimen Processing \\
\hline $\begin{array}{l}\text { 9. I feel competent collecting, labeling, } \\
\text { transporting, and processing } \\
\text { specimens for laboratory analysis. }\end{array}$ & Specimen Processing \\
\hline $\begin{array}{l}\text { 10. I feel competent to maintain a work } \\
\text { environment to enhance safety and } \\
\text { quality of laboratory procedures. }\end{array}$ & Workflow \\
\hline $\begin{array}{l}\text { 11. I feel competent contributing to the } \\
\text { efficient operation of the simulated } \\
\text { laboratory. }\end{array}$ & Workflow \\
\hline $\begin{array}{l}\text { 12. I feel competent following detailed } \\
\text { written instructions for performing } \\
\text { laboratory testing. }\end{array}$ & Workflow \\
\hline
\end{tabular}

\section{Modules}

Laboratory Data Entry. The students used different features of the LIS software as part of their weekly simulated laboratory sessions. Training review on the LIS system was provided in the form of a "dry laboratory" assignment the first week of Simulated Laboratory.

Specimen Processing. Students completed an online module, reviewing specimen processing then applied the learned concepts during the accompanying laboratory session.
Personnel Management and Communication Skills. During this section certain students were approached prior to the start of laboratory and asked if they would call in sick or leave part way through their shift. This forced laboratory managers to readjust workflow and personnel. In addition, the students assigned to be laboratory managers were also tasked with reviewing all laboratory results generated on their shift, checking for clerical and technical errors, confirming the accuracy of results, and ensuring that quality control and daily maintenance had been performed. Interpersonal issues with colleagues came up naturally, as they usually do in the real world, by student complaints regarding seemingly unfair workloads, and students not seeking out information in SOPs, but utilizing the MLS department lab manager. These complaints were treated as teaching moments and the students were encouraged to work through these issues with very little faculty input, other than some gentle facilitating of discussion.

The communication portion of the module focused on SBAR training, which is an acronym for Situation, Background, Assessment, Recommendation, a technique used to facilitate prompt and appropriate communication. This communication model has gained popularity in healthcare settings, especially among professions such as nursing. ${ }^{6}$ During the scheduled laboratory time, students were asked to call mock nurses or doctors with critical values and laboratory information vital to patient diagnosis and prompted to utilize the SBAR method of communication.

Workflow. This module focused on time management skills and adherence to turn-around-times. Students were encouraged to help with the workload of other departments when appropriate. STAT laboratory samples were introduced at this point and students were taught about reflex testing, working with short-draws, hemolyzed or lipemic samples, and performing hematology testing on sodium citrate tubes when EDTA samples were unavailable. The workload was varied throughout the semester, generally increasing throughout.

Instrument Maintenance, Troubleshooting, and Quality Control. This module consisted of monitoring temperatures in the laboratory, performing daily quality control for each workstation, preparation of reagents and calibration material, and performing scheduled 


\section{EDUCATION}

instrument maintenance.

Customer Service. This module covered topics as working well with colleagues, and patient confidentiality. Scenarios describing various customer service issues commonly encountered in a clinical laboratory were given as homework assignments and then discussed in class. In the laboratory, mock scenarios were developed to test the students' interaction with other healthcare workers and patients.

\section{Laboratory Setup Laboratory Schedule}

The Simulated Laboratory course required that each student perform ten phlebotomy procedures during the semester. Laboratory sessions were held on Monday and Tuesday afternoon. Each week faculty assigned two students as laboratory managers/specimen processors. These students arrived to the laboratory 20 minutes early and were responsible for scheduling each department with one or two students. They would proceed with accessioning and distributing all of the requisitions and samples for that day. Faculty purposely generated clerical errors such as mislabeled tubes, misspelled names, second identifier on tubes, missing tubes, ambiguous laboratory orders, incorrect sample types, multiple patients with the same last name and similar first names, and mislabeled hard to collect samples. The rest of the students populated each department as they arrived.

\section{Timeline}

The timeline for the laboratory preparation and operation is included in Table 2.

\section{Sample Prep}

Most laboratory sessions consisted of 4-5 Urinalyses, 810 CBCs with 3-5 manual differentials, 5-8 PT and aPTTs, 2-3 serology tests, a blood type with antibody screen, a cord blood sample, a crossmatch, 1-3 ESRs, 35 chemistry tests, and 5-10 gram stains. The gram stains were usually requested STAT in the middle of their workflow. The faculty labeled the samples, placed them into biohazard bags, and filled out requisition forms for each patient. Samples were delivered to the laboratory at different times throughout the two and a half hour session with the majority of samples given to the laboratory managers upon arriving to the laboratory.

\section{RESULTS}

\section{Statistical Analysis}

Comparison of the pre and post-mock certification exam was performed using a paired Student's t-Test and the $\mathrm{R}$ software package version 3.1.1. ${ }^{7}$ Descriptive statistics and graphical representations were also performed in $\mathrm{R}$ using the psych and ggplot2 packages. One individual did not take the post course survey or mock certification exam and was removed from the statistical analysis.

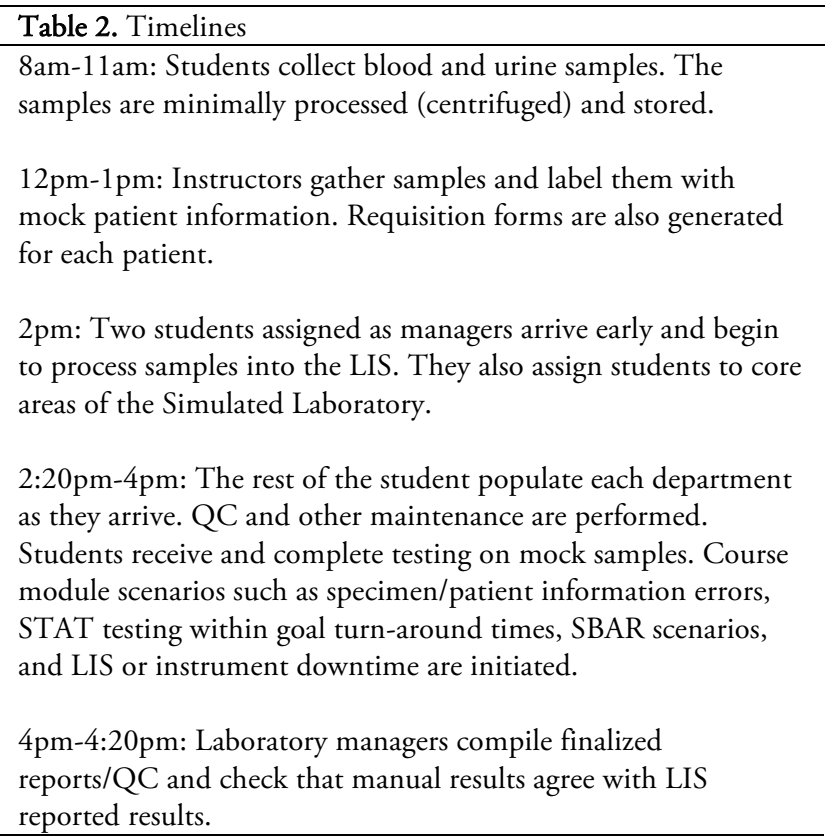

\section{Pre/Post Survey}

The students' perception and satisfaction with the course was evaluated using a twelve-question survey using a five point Likert scale response. The survey questions are shown in Table 1 and were designed to address six of the main course objectives: Laboratory Data Entry, Customer Service, Instrument Maintenance, Management and Communication, Specimen Processing, and Workflow. The results of the survey taken before (pre-) and after (post-) the students took the simulated laboratory course are shown in Figure 1. The two lowest scoring responses in the pre-survey show the students felt less competent in instrument maintenance and management and communication skills. The student post-survey reflected a lack of confidence regarding instrument maintenance; hence future Simulated Laboratory offerings will include more instrument troubleshooting. The highest scoring responses on the pre-survey were related to customer service and workflow. The mean response score for all the survey 


\section{EDUCATION}

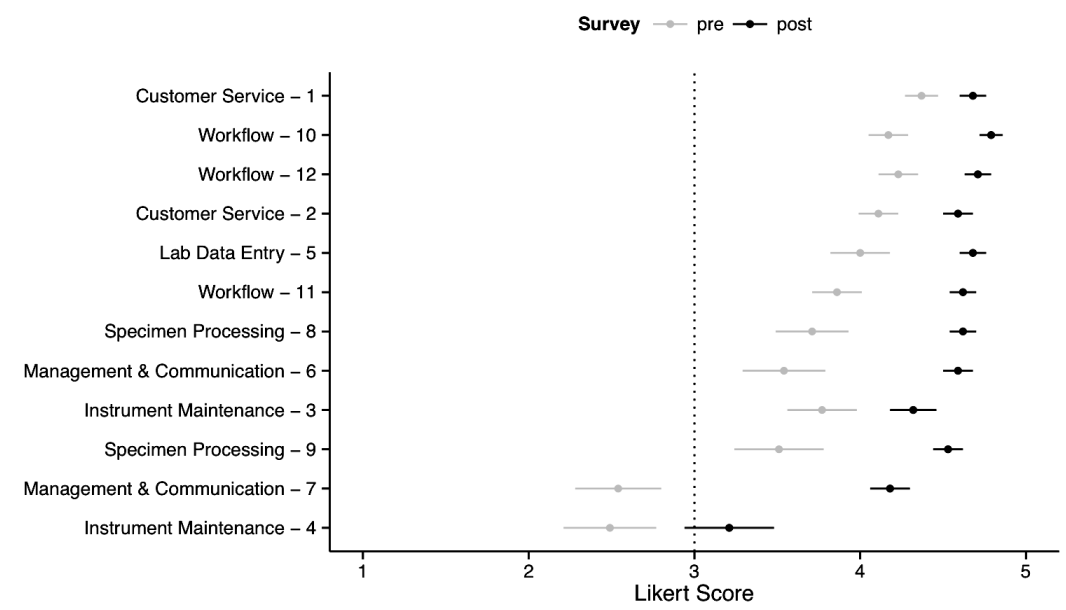

Figure 1. Student Course Survey Results. The Likert scores from the pre (grey) and post (black) surveys are shown with the mean score (dot) and $+/$ - one standard error of the mean (bar). Survey questions are shown on the $y$-axis with the course module and question number associated with Table 1. The combined mean score on both the pre and post survey ranks the questions. Likert Scores are defined as; 1 Strongly Disagree, 2 - Disagree, 3 - Neutral, 4 - Agree, and 5 - Strongly Agree. There was an N=35 for the pre survey results and an $\mathrm{N}=34$ for the post survey results over two semesters. The dotted line represents a response of neutral on the Likert scale.

questions increased in the post-survey. The greatest mean Likert score increase (1.64) was related to management and communication, "I feel competent providing teaching and education to laboratory students and other personnel".

\section{Pre/Post Certification Exam}

Students completed a 100-question mock Board of Certification (BOC) certification exam at the start of the Simulated Laboratory course. This score was used as a baseline and the results were not included in the student's final grade. After the completion of both semesters, the same mock certification exam was administered and compared to the pre course exam scores. The mean increase in percent grade on the mock BOC was 24.5 percentage points. (Figure 2.) This difference was found to be statistically significant $\left(\mathrm{p}=1.639 \times 10^{-7}\right)$ using a Student's paired t-Test.

\section{Final Practical Capstone}

The final capstone assessment exercise consisted of students working individually to complete five patient samples involving all of the major disciplines, including QC, specimen processing, and any instrument maintenance necessary within a pre-determined time frame of 90 minutes. All 18 students scored above $80 \%$ in this assessment.

\section{DISCUSSION}

A review of the literature has identified dwindling clinical rotation sites as a factor in limiting the number of medical laboratory science students a university-based program accepts. ${ }^{2}$ The Simulated Laboratory was created in response to this issue along with the assessment of WSU's advisory board and employer's requirements for skills that graduates possess. Through trial and error an effective learning environment was created to allow the students access to STAT situations while still being able to monitor them in the safety of an academic setting. By creating and implementing measurement tools such as surveys and mock certification exam scores, the faculty was able to assess the effectiveness of the Simulated Laboratory. These results and student evaluation results of the Simulated Laboratory courses have been favorable, suggesting that the course is meeting a need in MLS curriculum. Members of the advisory board, who felt that it wasn't enough to just perform patient testing in a STAT laboratory environment, suggested the capstone practical exam. They suggested that students should be subjected to a simulated environment where they had to work alone, completing all testing across all disciplines.

Anecdotal data include the fact that $100 \%$ of Simulated Laboratory graduates passed the BOC the first time in 2014. Of the 14 graduates to take the BOC in 2015 all have passed except one student, this student being the only student who did not take the mock postcertification exam. These data suggest that two semesters 


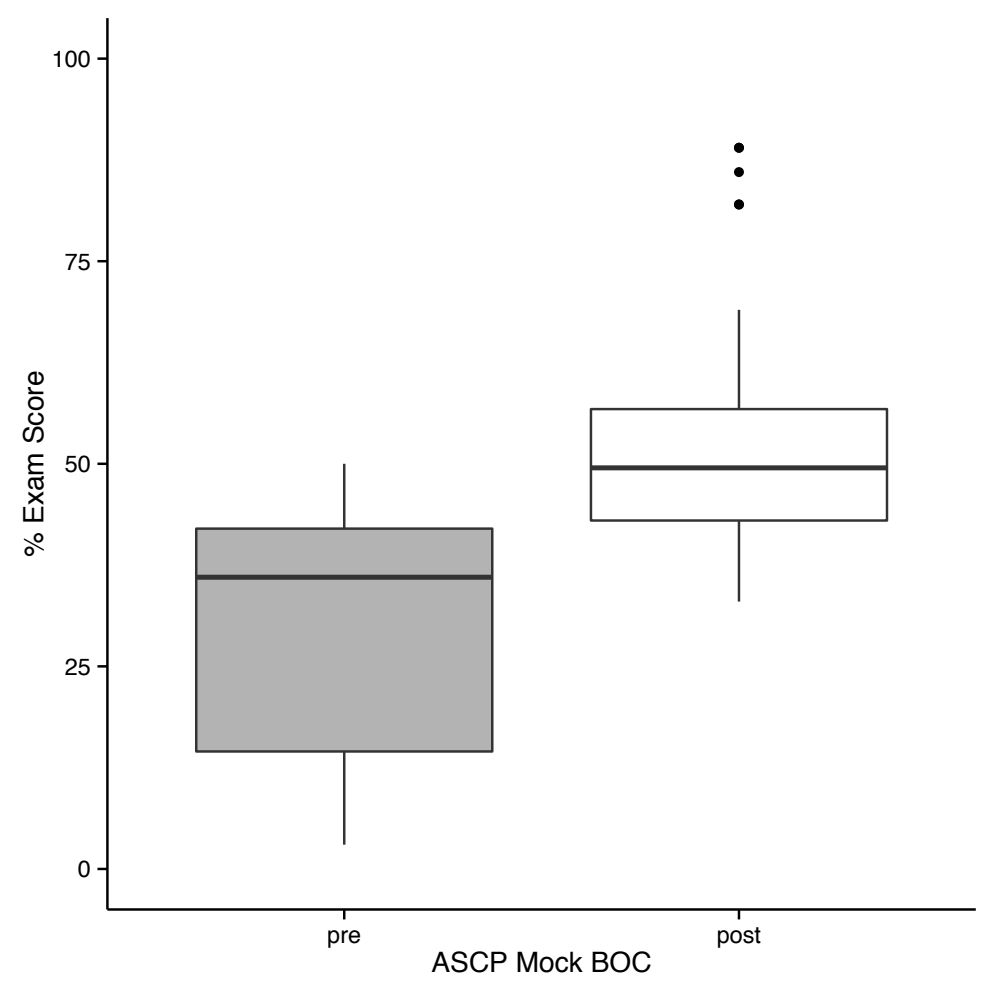

Figure 2. ASCP Mock Board of Certification Exam Scores. The pre and post ASCP mock MLS certification exam scores (N=34) are shown above in a box-and-whisker plot. The lower edge of the box represents the 25 percentile, the dark black line represents the median score, and the top edge of the box represents the 75 percentile. Outlying points fall beyond the whiskers that extend to the last point within $+/-1.5 \mathrm{x}$ the Inter Quartile Range. The paired increase in average score of 24.5 percent in the post compared to the pre exam was found to be statistically significant $\left(\mathrm{p}=1.639 \times 10^{-7}\right)$.

in the simulated STAT laboratory environment improved the students' generalist knowledge of MLS on average.

Some logistical problems were encountered during the development of the simulated laboratory. Donated instruments proved frustrating. The chemistry analyzer in particular had problems with ease of use, exasperating some of the students. The space that held the simulated STAT laboratory became too warm due to the increased amount of instrumentation. Purchasing a wall-mounted air conditioner for the room alleviated this issue. Another roadblock identified was the amount of preparation time required by the faculty each week. Between 2-3 hours were spent in the collection of samples, labeling, and creating requisitions weekly. This issue has yet to be resolved, although each semester does become easier to manage. Rescheduling the laboratory session to the middle of the week, as opposed to Mondays has helped alleviate some of the pressure with regards to preparation time. Overall students and faculty alike have received this course. The course has evolved and grown into an integral part of the department's clinical education as a capstone course for the BS level MLS students.

\section{Limitations}

A limitation of this study was the relatively small sample size of student participants. In addition, there are confounding factors such as students taking other core MLS courses during the same semester as Simulated Laboratory. Registration records were analyzed to investigate the latter which showed that increased post mock certification exam scores did not correlate with students taking core MLS courses during the same two semesters as Simulated Laboratory. (Figure 3) Continuing to collect data from future cohorts will assist in drawing more definite conclusions. 


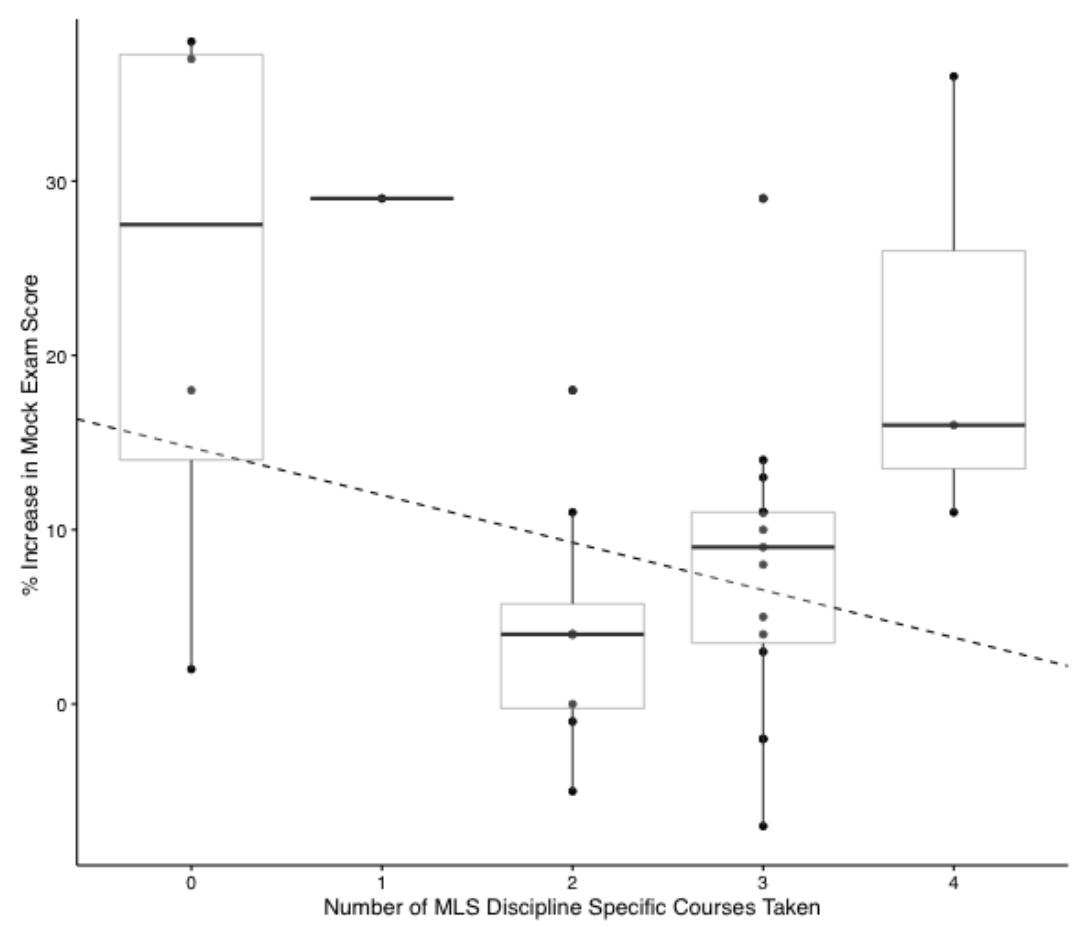

Figure 3. Percent Increase in Mock AsCL Certification Scores by the Numb er of MLS Discipline Specific Courses Taken. Vertical black lines show the median percent increase in the mock ASCP certification exam scores for each student group, which are defined by the number MLS disciple specific course that were taken during the simulated STAT laboratory. The dashed line represents a fitted linear regression model showing a non-significant negative trend.

\section{ACKNOWLEDGEMENT}

Special Thanks to: Scott Wright, MLS Department Chair, for his guidance and support in creating the simulated laboratory, Kent Criddle, MLS Lab Manager, for his help and support in maintaining the instrumentation and procuring the reagents and supplies necessary, Dr. Yasmen Simonian, the Dean of the Dumke College of Health Professions, for her tireless support of the MLS department, and Ryan Rowe, for his leadership in designing the simulated laboratory remodel.

\section{REFERENCES}

1. Tara K. What employers seek in job applicants: You've got the skills they want. [2013]. Available at <http://www.apa.org/ed/ precollege/psn/2013/09/job-applicants.aspx>

2. Bennett $A$, et al. Building a laboratory workforce to meet the future: ASCP task force on the laboratory professionals workforce. Am. J. Clin. Pathol. 2014;141:154-67.

3. Harr R. Clinical Laboratory Science Review Third Edition, 2007.

4. Ciulla A, Lehman D. Success in Clin Laboratory Science Fourth Edition, 2010

5. Board of Certification Study Guide Fifth Edition, 2009.

6. Leonard M, Graham S, Bonacum D. The human factor: the critical importance of effective teamwork and communication in providing safe care. BMJ Quality Safety Health Care 2004;13(Suppl 1):i85-i90. doi: 10.1136/qshc.2004.010033

7. Team R. D. C. R: A language and environment for statistical computing. [2008]. Available from <http://www.r-project.org> 